\title{
ATMOSPHERIC DEPTHS OF JUPITER, SATURN, AND URANUS
}

\author{
S. F. DER MOTT
}

The Royal Military College of Science, Shrivenham, Swindon, Wilts, England

\begin{abstract}
The presence of numerous near-commensurabilities among pairs of mean motions and the strong correlation between orbital radius and mass in the satellite systems of the three major planets (particularly in the Saturn system) suggest that the orbits of the satellites have evolved considerably under the action of tides. It is shown that the source of dissipation could be boundary layer turbulence at the base of the planetary atmosphere. If this is the source of dissipation then it should be possible to estimate the depths of these atmospheres from the mean rates of energy dissipation.
\end{abstract}

\section{Introduction}

It has been shown (Roy and Ovenden, 1954; Goldreich, 1965; Dermott, 1968) that the observed number of near-commensurate pairs of mean motions in the solar system is too great to have arisen from a random distribution of mean motions. Goldreich (1965) has shown that certain near-commensurabilities are stable and considers that the present non-random distribution of mean motions has been brought about by the action of tides. In this paper I indicate how Goldreich's tidal hypothesis can be tested. If it can be substantiated then a knowledge of the depths of the planetary atmospheres of Jupiter, Saturn, and Uranus may result.

The theory can be developed quantitatively and in substantial detail but as some parts of the argument have not yet been completed I consider a brief presentation to be the more appropriate.

\section{Simple Tide Theory}

In a satellite system which has been acted on considerably by tides there should be a strong correlation between orbital radius and mass. The rate of orbital evolution due to tidal action is mass dependent and thus in a satellite system in which initially there is no correlation between orbital radius and mass, tides will act causing the satellites to collide and grow until they are sorted according to their masses - the biggest satellite being the one furthest from the planet and the smallest the one nearest to it. This process may, of course, take some considerable time. The sorting process will also be checked to some extent by the formation of stable commensurabilities.

Consider the evolution of a single satellite. Standard theory shows that

$$
\frac{\mathrm{d} r}{\mathrm{~d} t}=f(p) \frac{m}{r^{11 / 2}} \cdot \frac{1}{Q}
$$

where $r$ is the orbital radius and $m$ the mass of the satellite, $f(p)$ is a function of parameters of the planet only and $Q^{-1}$ is the dissipative function (Dermott, 1968). It is

Sagan et al. (eds.), Planetary Atmospheres, 371-374.

All Rights Reserted. Copyright $: 1971$ by the I.A. $L$. 
probable that $Q$ is amplitude- and frequency-dependent, and the nature of this dependence must be determined before (1) can be integrated. As a first step I consider it reasonable to write

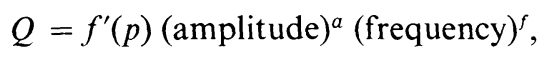

where $f^{\prime}(p)$ is a function of parameters of the planet only. Goldreich and Soter (1966) have shown that, if the source of dissipation is boundary layer turbulence at the base of the planetary atmosphere, then

$$
\left.\left.Q=f^{\prime}(p) \text { (amplitude) }\right)^{-1} \text { (frequency) }\right)^{-2} .
$$

The amplitude of the tide is proportional to $m / r^{3}$ and the frequency to $1-\left(r_{b} / r\right)^{3 / 2}$, where $r_{b}$ is the radius of the tidal barrier (see Dermott (1968)). As $r_{b} \ll r$, for most satellites, the frequency term can be neglected in the first instance and we can write

$$
Q=f^{\prime}(p) r^{-3 a} m^{a} .
$$

On substitution of this expression into (1) and integration we obtain

$$
r^{13 / 2-3 a}\left\{1-\left(\frac{r_{i}}{r}\right)^{13 / 2-3 a}\right\}=f^{\prime \prime}(p) m^{1-a} t,
$$

where $r_{i}$ is the initial orbital radius and $t$ the time of evolution. We have $a \leqslant 0(Q \rightarrow \infty$ as amplitude $\rightarrow 0$ ) and therefore $\left(r_{i} / r\right)^{13 / 2-3 a} \ll 1$ and can be neglected if evolution has been appreciable. Thus,

$$
\log r=\frac{1-a}{13 / 2-3 a} \log m+\frac{\log f^{\prime \prime}(p) t}{13 / 2-3 a}
$$

Thus the orbital radius of a satellite which has been acted on appreciably by tides is virtually independent of its initial orbital radius. If we allow that in any one system all the satellites are of one age and that the evolution of a single satellite can be treated independently of all the others, then we can predict that in a tidally evolved satellite system there will be a linear correlation between $\log r$ and $\log m$.

A plot of $\log r$ against visual magnitude for the satellite system of Uranus is shown in Figure 1 (the masses of these satellites are not known). A plot of $\log r$ against $\log m$ for the satellite system of Saturn is shown in Figure 2. The line in the latter diagram is drawn to pass through Titan and has a slope corresponding to $a=-1$. The correlation coefficients of the two plots are respectively 0.005 and 0.001 . I take this to be strong supporting evidence that the satellite systems of Saturn and Uranus are tidally evolved systems. The satellite system of Jupiter cannot be treated in the above manner as all of the four major satellites are linked together in a presumably stable manner and thus cannot possibly be treated separately. The very presence of the numerous near-commensurabilities in this system though indicates that it also is a tidally evolved system.

A fuller theory is needed (and has in part been developed) to take account of: (a) the frequency dependence of $Q$; (b) the formation and evolution of stable commensurabilities; and (c) satellites of varying ages, but the most interesting feature of a tidally 


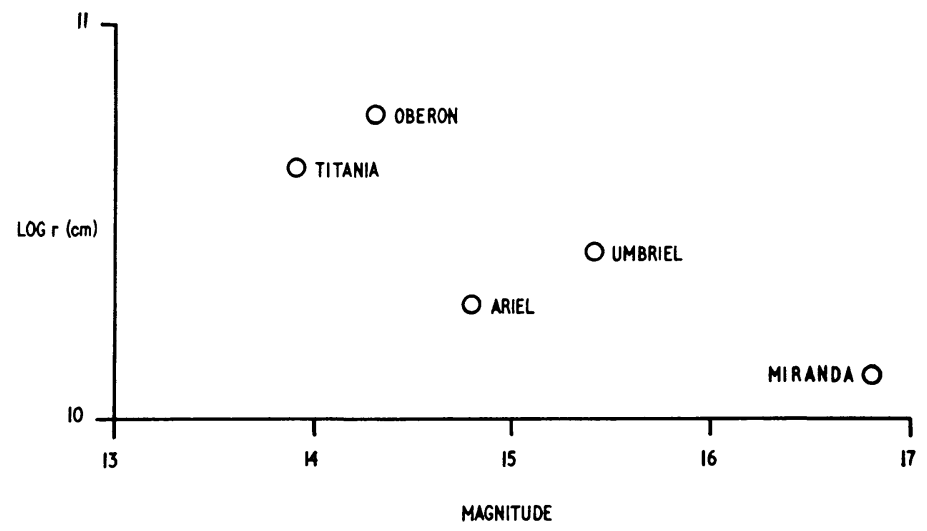

Fig. 1. A plot of log of orbital radius against visual magnitude for the satellite system of Uranus.

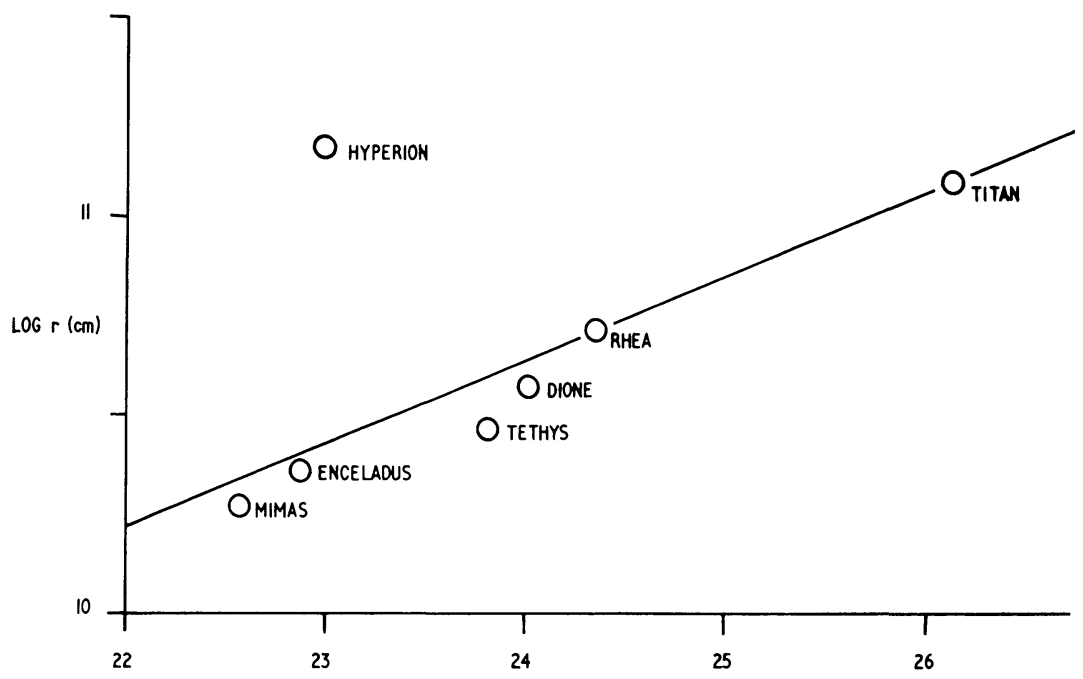

Fig. 2. A plot of $\log$ of orbital radius against $\log$ of mass for the satellite system of Saturn.

evolved satellite system has been revealed by the simplified discussion given above - the present orbital radius of a tidally evolved satellite does not depend on its initial orbital radius. Because of this it should be possible to correlate the orbital radii and the masses of the satellites in any one system in an exact manner, e.g. as the mass of Titan is known to three figures it should be possible to predict that of Rhea to three figures.

\section{Conclusion}

The features of the satellite systems of the major planets which suggest they are tidally evolved are: 
(i) the preference for near-commensurability among pairs of mean motions (Jupiter and Saturn),

(ii) the linear correlation between $\log r$ and $\log m$ (Saturn and Uranus).

It would appear that in the Saturn system $Q$ is inversely proportional to amplitude and thus the source of dissipation could be boundary layer turbulence at the base of the planetary atmosphere. If we assume that this is the source of dissipation on Jupiter and Uranus as well as Saturn then it should be possible to estimate parameters associated with the depths and density structures of these atmospheres from the mean rates of energy dissipation (this being related to $f^{\prime \prime}(p)$ in (2)). It would then have to be shown, of course, that the estimates were in reasonable accord with our present (somewhat limited) knowledge of the structure of these planets and that no other possible source of dissipation could account for the observations.

Perhaps at this stage it is best simply to state that the consequences of tidal evolution can and should be determined in a detailed quantitative manner and that if the predictions of the theory are substantiated then some knowledge of the nature of the major planets should result.

\section{References}

Dermott, S. F.: 1968, Monthly Notices Roy. Astron. Soc. 141, 349.

Goldreich, P.: 1965, Monthly Notices Roy. Astron. Soc. 130, 159.

Goldreich, P. and Soter, S.: 1966, Icarus 5, 375.

Roy, A. E. and Ovenden, M. W.: 1954, Monthly Notices Roy. Astron. Soc. 114, 232. 